\title{
Blepharoconjunctivitis Associated to Infraorbital Sinuses Granuloma in a Cockatiel (Nymphicus hollandicus)
}

\author{
Marco Di Giuseppe $^{1 *}$, Chiara Pante ${ }^{2}$, Danilo Grande ${ }^{3}$, Manuel Morici ${ }^{4}$ \\ ${ }^{1}$ Centro Veterinario per Animali Esotici, Palermo, Italy \\ ${ }^{2}$ Freelance Ophthalmologist Veterinarian, Palermo, Italy \\ ${ }^{3}$ Centro Veterinario Villa Flaviana, Roma
}

${ }^{4}$ Safari Park, Pombia, Novara, Italy

Received: 17 July, 2017; Accepted: 21 August, 2017; Published: 24 August, 2017

*Corresponding author: Marco Di Giuseppe,Centro Veterinario per Animali Esotici, Palermo, Italy; E-mail: marcodigiuseppe@yahoo.com

\section{Abstract}

This case report describes a blepharoconjunctivitis associated with an infraorbital sinus granuloma in a 7 years female cockatiel. The animal was referred for a bilateral conjunctivitis, initially treated with a medical therapy that produced no improvement of clinical signs because of the presence of a suborbital mass. During the exploratory surgery the mass was compatible with a granuloma of infraorbital sinus. Unlucky, the recurrence of this granuloma needed some diagnostic exams to isolate the c cause of the mass and the correlated infection. The aim of this article is to underline the importance of a correct diagnostic approach and the need of a combination of a specific pharmacological treatment with a surgery to solve such a problem even if literature seems to suggest mainly a medical approach.

Keywords: Granuloma; Psittacine; Cockatiel; Sinusitis; Infraorbital Sinus; Supraorbital mass

\section{Clinical case}

A 7 years old female cockatiel was referred to Veterinary hospital "Città di Palermo" Palermo (Italy), where first author have been worked as referral veterinarian for exotic animal, because of bilateral blephaconjunctivitis since one week and anorexia for few days.

According to its medical history, its diet was based only on sunflower seeds.

At the clinical examination, the parrot was cachectic and its feathers appeared in very bad condition. The periocular area was alopecic and where present, feathers were wet and damaged due to epiphora and scratching. Moreover Blepharospasm, eyelid oedema, epiphora and a thick lump in the supraorbital part of both eyes were noticed (Figure 1).

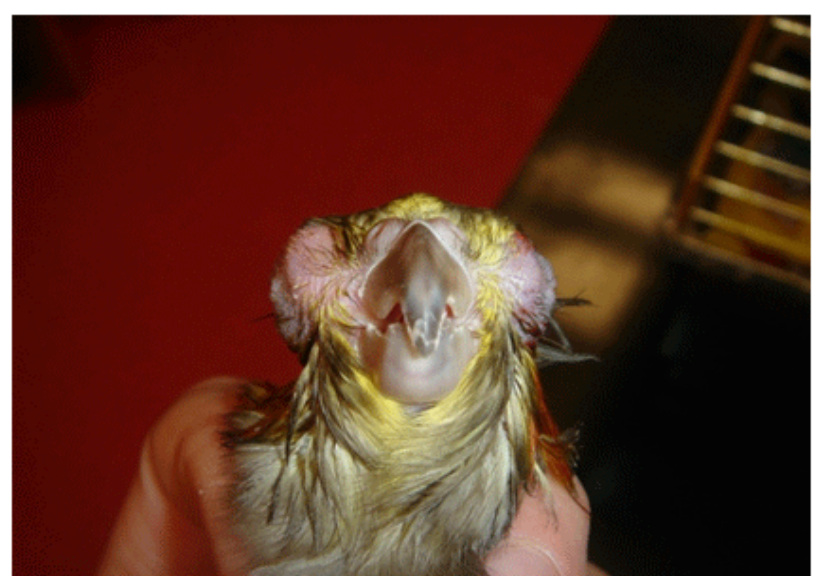

Figure 1: Cockatiel with blepharoconjunctivitis associated with swollen eye during its first visit. The eye are so swollen that cockatiel wasn't able to opening the eyelids
Application of fluorescein in the conjunctival sac didn't reveal any ulcera as well as occlusion of the lacrimal duct. From the crop was isolated Trichomonas sp. while from the conjunctiva was isolated Pseudomonas Aueruginosa sensitive to Amikacin, Piperacillin and Ceftazidime.

Trichomonas $s p$, although is a common finding in the gastroenteric system, is able to spread in the air sacs thought the choanes [5].

Based on clinical and laboratory findings the animal was treated with:

- metronidazole $25 \mathrm{mg} / \mathrm{kg}$ twice a day for 3 weeks to treat trichomoniasis, -ceftazidime IM a $100 \mathrm{mg} / \mathrm{kg}$ q8hours for 3 weeks according to the colture and sensitivity test,

-tobramycin ophthalmic solution 1 drop q8hours for 2 weeks,

-piroxicam ophthalmic solution 1 drop twice a day for 2 weeks,

-washing of nostrils and infraorbital sinuses and aerosol with thiamphenicol glycinate acetylcysteine in order to fluidify the secretions responsible for the lacrimal duct obstruction twice a week for 2 weeks.

Based on clinical suspects the animal was treated with:

-doxycycline $50 \mathrm{mg} / \mathrm{kg}$ q7die for 4 weeks for a suspected Chlamydophila psittaci infection as the owner doesn't allow to test for it.

-vitamin A $2000 \mathrm{UI} / / \mathrm{kg}$ orally for 10 days for a suspected hypovitaminosis due to wrong diet. Moreover a pellet diet was introduced.

After two weeks of treatment, the cockatiel was feeding by its own with pellet diet gaining weight but the swelling of the eyes were still present. Therefore an explorative surgery was performed to determine the origin of such swelling.

Induction of anesthesia was performed with midazolam $0.1 \mathrm{mg} /$ $\mathrm{kg}$ and butorphanole $1 \mathrm{mg} / \mathrm{kg}$ injection in pectoral muscles and once intubated, the anesthesia was maintained by a mixture of oxygen $0.5 \mathrm{~L} /$ min and $2 \%$ isoflurane.

The skin was disinfected with $4 \%$ chlorhexidine and a $1 \mathrm{~cm}$ incision was performed on both supraorbital masses on the dorsal surface of the upper eyelid.

Under the incisions the presence of a peanut sized granuloma were reveled. Surgical incisions were left open to allow further washings as well as to grant drainage (Figure 2,3). 


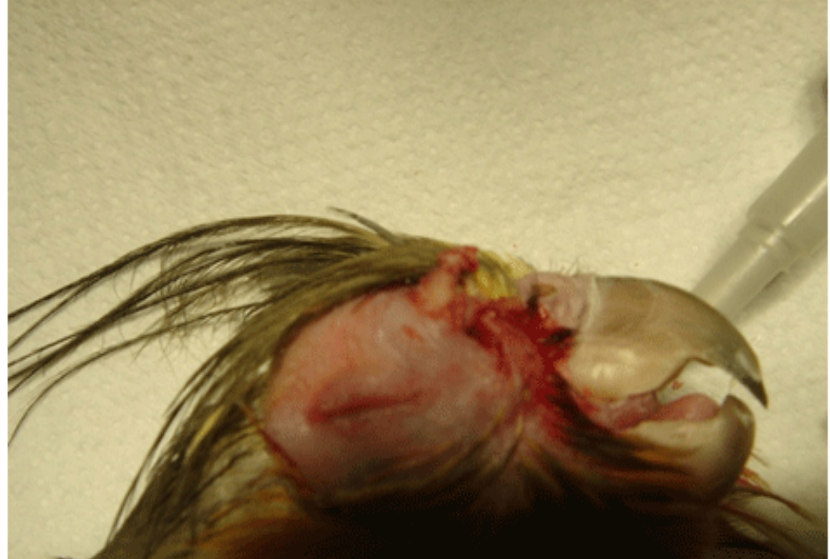

Figure 2: Medical treatment of blepharoconjunctivitis didn't improve the clinical presentation. For this reason an exploratory surgery of the infraorbital sinus was performed: incision of the dorsal aspect of the upper eyelid on the supraorbital mass showing above a granuloma of the infraorbital sinus

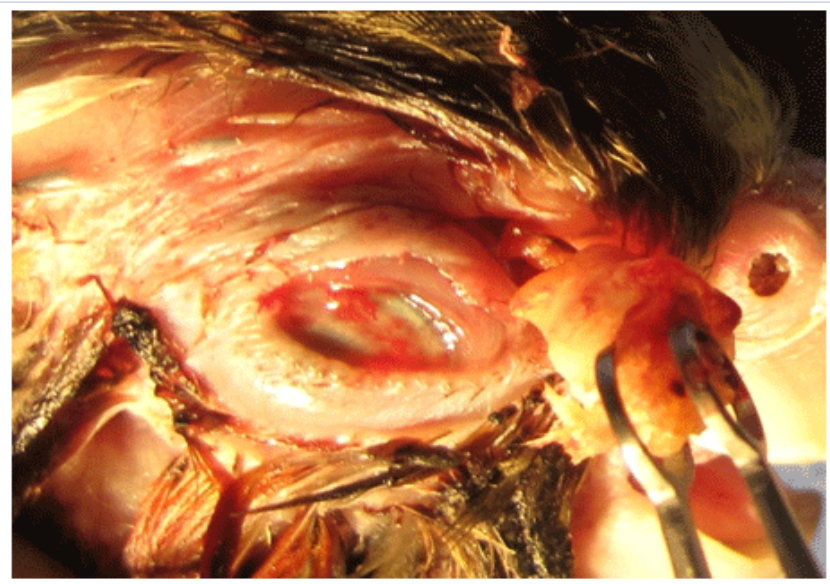

Figure 3: Particular of the granuloma removed from the right infraorbital sinus

Owner didn't allow sending granulomas for a culture and sensibility test.

The parrot was discharged with meloxicam $0.2 \mathrm{mg} / \mathrm{kg}$ twice a day for 5 days to control postoperative swelling as well as postoperative pain. Metronidazole and ceftazidime were continued for 1 week more to finish the initial therapy. Doxiciline was administered 2 times more to reach 1 month of therapy that is the minimum therapeutic time needed to treat Chlamydiophila psittaci being an obligated intracellular bacteria.

At the post-operative checkup, after 14 days, all the medical therapy was ended. The left eye was completely recovered while the right eye appeared still swollen with a protrusion of the third gland (Figure 4,5). Another exploratory surgery was performed in the right eye reveled a recurrences of the granuloma. At this point owner allow to send granuloma removed for a culture and sensitivity test. Results showed the presence of Streptococcus Aureus, sensitive only to enrofloxacin.

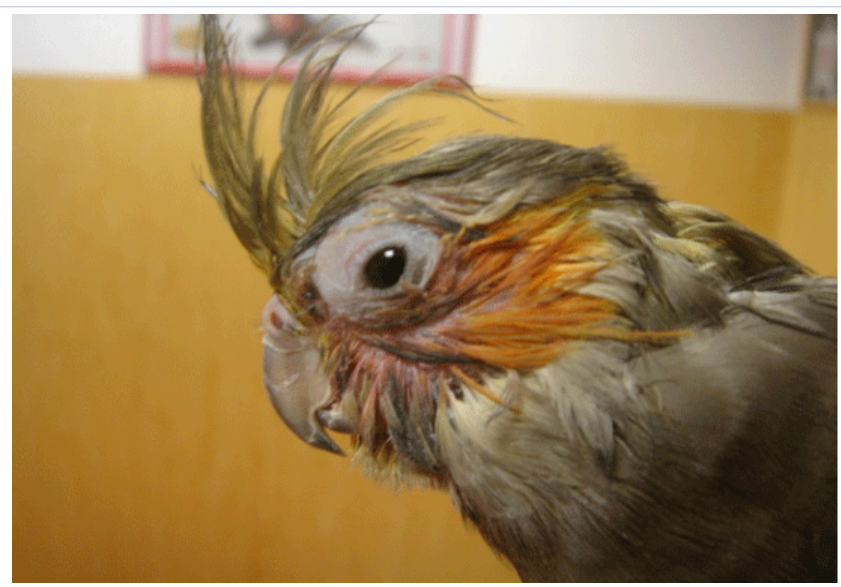

Figure 4: Left eye at the first postoperative checkup. The eye is no more swollen but an epiphora is still present as the feathers around the eye are wet

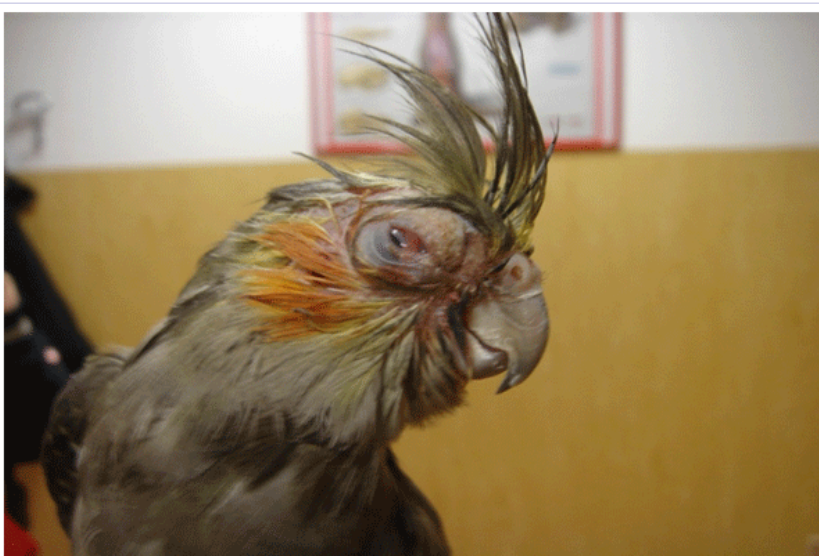

Figure 5: Right eye at the postoperative checkup. Although the eye is less swollen than the first presentation the upper eyelid appear still swollen

Again, the cockatiel was discharged with meloxicam for postoperative swollen and pain and based on culture and sensitivity result enrofloxacin $10 \mathrm{mg} / \mathrm{kg}$ twice a day for 2 weeks was added.

At the end of the therapy postoperative check up revealed the righ eye completely recovered with the surgical incision still open and the left one in top condition (Figure 6,7).

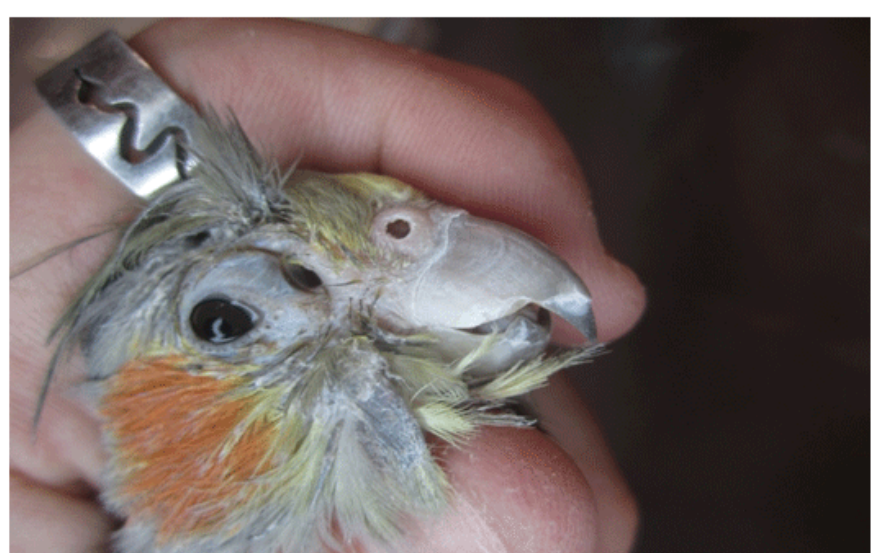

Figure 6: Right eye at the postoperative checkup after the second surgery. The incision was left open to allow the wound to drain and help the clinician to medicate it

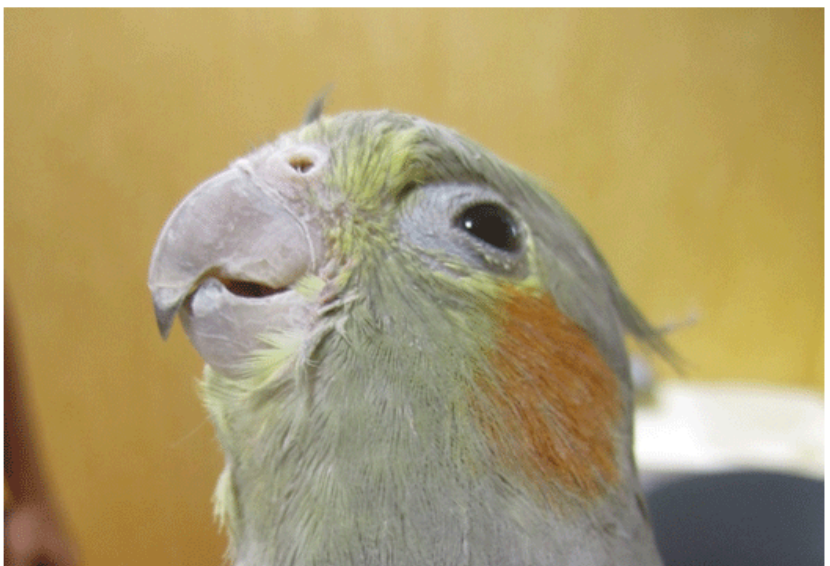

Figure 7: Left eye in top condition at the end of the treatment

\section{Discussion}

According to the authors' opinion, the recurrence of the granuloma should be connected to its incomplete removal during the first surgery as well as to the impossibility to isolate the etiological agent. Consequently, the antibiotic therapy was based only on the result of the conjunctival culture and sensitivity test that could be only a secondary infection.

Moreover, the multi-resistance of Pseudomonas Aueruginosa isolated from the conjunctiva and of Streptococcus Aureus isolated from the granuloma underline the chronicization of both the infection process.

Although in psittacids blepharoconjunctivitis associated to sinusitises 
respond well to specific medical treatments, in the case reported the presence of a granuloma in both the infraorbital sinuses did not allow to solve the problem with only pharmacological treatment and washings of the infraorbital sinuses $[3,4]$.

Although the animal size can hamper surgery on the particular area the authors state that such surgery represents the main solution to adopt, as the nature of the granuloma does not allow a less invasive approaches.

However, it is important to consider that during surgery an excessive bleeding must be prevented and that during washings particular attention must be paid because of the direct connection of the infraorbital sinuses to the nasal cavities, as it is possible to accidentally cause an ab-ingestis pneumonia [6].

A correct tier is mandatory in order to identify the causes and guaranteeing therapeutic success during the postoperative period.

However, it is also important to underline that in psittacids a diet based only on sunflower seeds associated to ocular problems should always orient the clinician at a hypovitaminosis A $[1,2,3,4]$ as well as to an aspergillosis. In fact the aspergillus sp. often settles on the seeds cuticle and can be inhaled through the choanas when the parrot tries to peel the seed. In this case, the culture did not show any fungal growth [7].

\section{References}

1. Doneley B. Avian medicine and surgery in practice. Manson Publishing. 2009;135-136, 185-186.

2. Pitts C. Hypovitaminosis A in psittacines. In: Kirk RW editor. Current Veterinary Therapy VIII. Philadelphia, WB, Saunders Co;1985;622-625.

3. Gelatt KN. Veterinary Ophthalmology. 4th ed. Blackwell Publishing; 2007;1381-1387.

4. Abrams GA, Murphy JP, Murphy CJ. Conjunctivitis in birds. Vet Clin North Am Exot Anim Pract. 2002;5(2):287-309.

5. Garner MM, Sturtevant FC. Trichomoniasis in a blue-fronted Amazon parrot (Amazona aestiva); J Assoc Avian Vet 1992;6:17-20.

6. Coles BH. Essentials of Avian medicine and Surgery. 3th ed. Blackwell Publishing; 2007;145-146.

7. McMilian MC, Petrak ML. Aspergillosis in pet birds: A review of 45 cases. Proc Assoc Avian Vet. 1988;35-45 